\title{
The pharmacogenetics of metformin
}

\author{
Jose C. Florez ${ }^{1,2,3,4,5}$
}

Received: 20 March 2017 / Accepted: 12 May 2017 /Published online: 3 August 2017

(C) Springer-Verlag GmbH Germany 2017

\begin{abstract}
Despite its widespread use as the first-line agent for the treatment of type 2 diabetes, it has become clear that metformin does not work optimally for everyone. Elucidating who are the likely metformin responders and non-responders is hampered by our limited knowledge of its precise molecular mechanism of action. One approach to achieve the related goals of stratifying patients into response subgroups and identifying the molecular targets of metformin involves the deployment of agnostic genome-wide approaches in cohorts of appropriate size to attain sufficient statistical power. While candidate gene studies have shed some light on the role of genetic variation in influencing metformin response, genome-wide association studies are beginning to provide additional insight that is unconstrained by prior knowledge. To fully realise their potential, much larger samples need to be assembled via international collaboration, preferably involving the academic community, government and the pharmaceutical industry.
\end{abstract}

Electronic supplementary material The online version of this article (doi:10.1007/s00125-017-4335-y) contains a slideset of the figures for download, which is available to authorised users.

Jose C. Florez

jcflorez@partners.org

1 Diabetes Unit, Massachusetts General Hospital, Boston, MA, USA

2 Center for Genomic Medicine, Simches Research Building-CPZN 5.250, Massachusetts General Hospital, 185 Cambridge Street, Boston, MA 02114, USA

3 Metabolism Program, Broad Institute, Cambridge, MA, USA

4 Program in Medical and Population Genetics, Broad Institute, Cambridge, MA, USA

5 Department of Medicine, Harvard Medical School, Boston, MA, USA
Keywords Genome-wide association studies · Metformin · Pharmacogenetics $\cdot$ Review $\cdot$ Single nucleotide polymorphisms · Type 2 diabetes

\begin{tabular}{|c|c|}
\hline \multicolumn{2}{|c|}{ Abbreviations } \\
\hline DPP & Diabetes Prevention Program \\
\hline GoDARTS & $\begin{array}{l}\text { Genetics of Diabetes and Audit Research } \\
\text { Tayside Study }\end{array}$ \\
\hline GWAS & Genome-wide association studies \\
\hline MATE & Multidrug toxin and extrusion \\
\hline MetGen & Metformin Genetics (Consortium) \\
\hline OCT & Organic cation transporter \\
\hline PMAT & Plasma monoamine transporter \\
\hline UKPDS & UK Prospective Diabetes Study \\
\hline
\end{tabular}

\section{The problem}

Metformin is the undisputed queen among type 2 diabetes drugs. She is enthroned atop all professional society algorithms as the first-line drug to be prescribed as soon as the diagnosis is made [1-3], in the absence of contraindications such as severe renal or hepatic insufficiency. This is because among the 12 available drug classes for type 2 diabetes, it alone embodies the three cardinal virtues expected of good medicines: it is eminently safe, cheap and effective $[4,5]$. Indeed, metformin therapy is well tolerated, with mild gastrointestinal side effects in $\sim 30 \%$ of patients; only $\sim 5 \%$ of users stop medication because of severe intolerance [6, 7]. In general, metformin is highly efficacious and is one of the few type 2 diabetes oral agents that promote weight loss rather than weight gain $[6,8,9]$. In addition, this weight loss is particularly beneficial and similar to exercise-induced weight loss, in that it does not reduce resting energy expenditure [10]; 
it is also sustained and dependent on adherence [11]. Thus, it has become one of the most commonly used medications for type 2 diabetes worldwide, with more than 48 million prescriptions filled in the USA in 2010. As described elsewhere in this issue of Diabetologia, metformin is also used for diabetes prevention [12] and the treatment of polycystic ovary syndrome [13], and there is hope that its use will expand to other indications, such as cancer treatment and/or prevention [14].

Despite all of these favourable qualities, metformin is not a panacea. Clinical practice suggests, and published trials confirm, that metformin monotherapy is often insufficient in achieving glycaemic control. Very frequently, escalation of therapy is required [15] and as many as $21 \%$ of patients initiated on metformin treatment fail to meet glycaemic goals in the first 5 years of therapy [16]. The failure rate exceeds $50 \%$ in youth with newly diagnosed type 2 diabetes, a group in whom diabetes incidence is rising at alarming rates [17]. The long-term follow-up of the Diabetes Prevention Program (DPP) showed that participants randomised to the effective metformin preventive intervention group continued to develop diabetes over time, even while on treatment and despite an adherence rate near $80 \%$, heightening the concern that metformin may only delay (by about 12 months on average), rather than prevent, diabetes incidence [9].

The metformin scenario thus illustrates a key barrier in modern therapeutics: by focusing on the 'average patient', as typified by the ideal responder in a successful clinical trial, we neglect to capture and describe the full range of pharmacological responses and thereby fail to identify those in whom a specific therapy might not be appropriate. Precious time might be wasted in trying to achieve glycaemic control in people in whom such a goal might be unreachable, and some patients may be unnecessarily exposed to an unacceptable level of side effects. To realise the promise of 'precision medicine' we need to transcend the law of averages and begin to characterise the subgroup of patients who would benefit from an alternative to metformin upfront, either because they do not tolerate it or because they fail to achieve glycaemic targets.

Why are we so limited in our ability to define a group of metformin non-responders a priori? In part, this is due to our ignorance around the molecular target of metformin and its precise mechanism of action. While its physiological effects in humans have been described and we are gaining new insights on the cellular pathways affected by the drug [18], we are still unclear as to the specific molecular entity that metformin physically engages with to modulate metabolism. This knowledge gap hampers the design of experiments aiming to describe likely non-responders but it also presents an opportunity to conduct agnostic screens that might yield fundamental discovery.

\section{The genetic approach as a potential solution}

Some of the individual differences that underlie the variation in response to metformin are likely genetic in nature. For example, epidemiological studies suggest that ethnic disparities exist in metformin response [19], and a genetic component is likely to underlie some of these disparities. Nevertheless, environmental factors may also be at play, either directly or by interacting with the genetic background; to guide further exploration it would be useful to quantify the relative contribution of each. Typically, this is done via classical heritability studies but these present obvious challenges in finding sufficient numbers of close relatives treated with metformin and phenotyped for therapeutic response. More recently, the availability of dense genome-wide genotyping in cohorts in which metformin response has been quantified allows investigators to measure the degree of genetic relatedness with adequate precision and estimate its contribution to the variance in the trait of interest [20].

The Genetics of Diabetes and Audit Research Tayside Study (GoDARTS) research group has pioneered genomewide association studies (GWAS) for metformin response in participants with type 2 diabetes [21]. GoDARTS has enrolled over 17,000 patients with established type 2 diabetes, followed in their clinical system, and matching non-diabetes control participants for genetic studies, leveraging the clinical information available in the electronic medical record. Most of these participants have undergone genome-wide genotyping and investigators have defined various measures of glycaemic metformin response based on the level of $\mathrm{HbA}_{1 \mathrm{c}}$ at the time of initial metformin prescription as well as its change while on treatment. They have also been able to control for adherence by accessing pharmacy records. Using the genome-wide complex trait analysis method [22], Zhou et al analysed 2085 participants with available data and concluded that the heritability of metformin response based on common variants captured by commercial genotyping arrays ranged from 20 to $34 \%$, depending on the specific measure examined, providing quantitative boundaries for the genetic effect, at least as far as variants shared across populations are concerned (Table 1) [23]. Heritability could possibly be higher if less common variants are also considered.

Having established that searching for genetic determinants of metformin response is sensible, it becomes worthwhile to articulate the main goals of pharmacogenetic inquiry (see Text box) [24]. One goal is to use genetic variation to stratify patients into likely responders and non-responders, so as to tailor therapy to those most likely to benefit. A related but distinct goal is to identify genes that encode putative drug targets, particularly for those pharmacological agents for which mechanism of action is still obscure. A third goal involves the use of a known pharmacological perturbation to 
Table 1 Heritability estimates of various measures of glycaemic response to metformin obtained from a genome-wide association study in GoDARTS

\begin{tabular}{ll}
\hline Glycaemic measure & Heritability $\left(h^{2}\right)$ \\
\hline Absolute reduction in $\mathrm{HbA}_{1 \mathrm{c}}{ }^{\mathrm{a}}$ & $23 \%$ \\
Proportional reduction in $\mathrm{HbA}_{1 \mathrm{c}}{ }^{\mathrm{b}}$ & $20 \%$ \\
Adjusted reduction in $\mathrm{HbA}_{1 \mathrm{c}}{ }^{\mathrm{c}}$ & $34 \%$ \\
Achieved target $\mathrm{HbA}_{1 \mathrm{c}}{ }^{\mathrm{d}}$ & $32 \%$ \\
\hline
\end{tabular}

The genome-wide complex trait analysis method [22] was deployed in 2085 GoDARTS participants to estimate heritability $\left(h^{2}\right)$ of various $\mathrm{HbA}_{1 \mathrm{c}}$-based measures of metformin response. Baseline $\mathrm{HbA}_{1 \mathrm{c}}$ was obtained within 6 months of metformin initiation and on-treatment $\mathrm{HbA}_{1 \mathrm{c}}$ was defined as the lowest value attained within 18 months of metformin initiation

${ }^{a}$ Difference between baseline and on-treatment $\mathrm{HbA}_{1 \mathrm{c}}$

${ }^{\mathrm{b}}$ Absolute reduction in $\mathrm{HbA}_{1 \mathrm{c}}$ divided by baseline $\mathrm{HbA}_{1 \mathrm{c}}$

${ }^{\mathrm{c}}$ The residuals of absolute reduction in $\mathrm{HbA}_{1 \mathrm{c}}$ adjusted by relevant clinical covariates, such as baseline $\mathrm{HbA}_{1 \mathrm{c}}$, patient adherence, metformin dose, renal function as estimated by creatinine clearance and treatment group

${ }^{\mathrm{d}}$ Categorical phenotype of whether or not on-treatment $\mathrm{HbA}_{1 \mathrm{c}}$ reached the intended target of $<7 \%$, after adjustment for baseline $\mathrm{HbA}_{1 \mathrm{c}}$ and relevant clinical covariates

Table adapted from [23]

illuminate the role of a gene of unknown function that encodes variation responsible for the differential response; however, for this latter exercise to be fully informative the precise mechanism of action of the drug must be understood for the gene product to be placed in the relevant pathway, which is not yet the case for metformin. Thus, genetic inquiry around metformin action has initially focused on the twin goals of stratifying patients by levels of response and identifying its molecular targets.

\section{Aims of pharmacogenetic studies}

(1) Patient stratification Genetic data may be used to categorise individuals into subgroups based on clinical response to the drug of interest

2 Target identification Agnostic genome-wide studies may identify genes that encode drug targets, elucidating a drug's mechanism of action and enabling the design of novel drugs that act on the same pathway

3 Functional characterisation Because drugs perturb the human organism in vivo, detecting a differential response based on a given genetic variant may illuminate the function of the gene product encoded by the gene that harbours the variant, or whose expression is influenced by it
Prior to the development of GWAS approaches to interrogate the entire genome, investigators were limited by pre-existing biological knowledge; for metformin, research had centred on the cellular transporters that controlled its metabolism, which could now be queried via candidate gene association studies. If robust associations were found they might subserve the classification of patients into responders and non-responders, but no new insights on metformin's targets would be gained; the panorama would be radically altered with the advent of GWAS. The next sections describe these efforts chronologically.

\section{First phase: candidate gene studies}

As a hydrophilic molecule, metformin cannot traverse cell membranes easily and is actively transported via organic cation transporters in enterocytes, hepatocytes and renal epithelial cells (Fig. 1) [25]. After oral ingestion, metformin is taken up by enterocytes via the plasma monoamine transporter (PMAT; encoded by the gene SLC29A4) and the organic cation transporter (OCT) 3 (encoded by SCL22A3), both of which are localised to the luminal surface of the gut epithelium [26, 27]. Transport to the serosal side occurs via OCT1 (encoded by SLC22A1) [27]. In the liver, both OCT1 and OCT3 seem to be responsible for metformin uptake into hepatocytes [25, 27-29], though, at least in mice, OCT1 appears to be the main driver [30]. Metformin is disposed into bile by the multidrug toxin and extrusion (MATE) 1 (encoded by $S L C 47 A 1)$ [31, 32], though in humans the bulk of metformin is actively excreted unchanged into the urine [25]: OCT2 (encoded by SLC22A2) takes it up into renal epithelial cells through the basolateral membrane [33], while MATE1 and MATE2 (the latter encoded by SLC47A2) excrete it into the urine $[31,32,34]$. The expression of OCT1 and PMAT on the apical membranes of renal epithelial cells suggests that these transporters may also mediate metformin reabsorption [35].

The most studied transporter regarding the impact of genetic variation on metformin action has been OCT1. The gene encoding OCT1, SLC22A1, is highly polymorphic, with a number of coding missense SNPs that affect its activity [36-39]. The Giacomini group pioneered some of these studies, showing that the presence of at least one of four reducedfunction variants (R61C/rs12208357, G401S/rs34130495, M420del/rs72552763 and/or G465R/rs34059508) attenuates the glycaemic effects of a short course of metformin [30], likely by an impairment of intracellular transport resulting in higher maximal and AUC plasma concentrations [40]. However, in a large retrospective observational clinical cohort of 1531 individuals assembled by GoDARTS, the two most common reduced-function SNPs (R61C and M420del) were not associated with four different measures of metformin glycaemic 

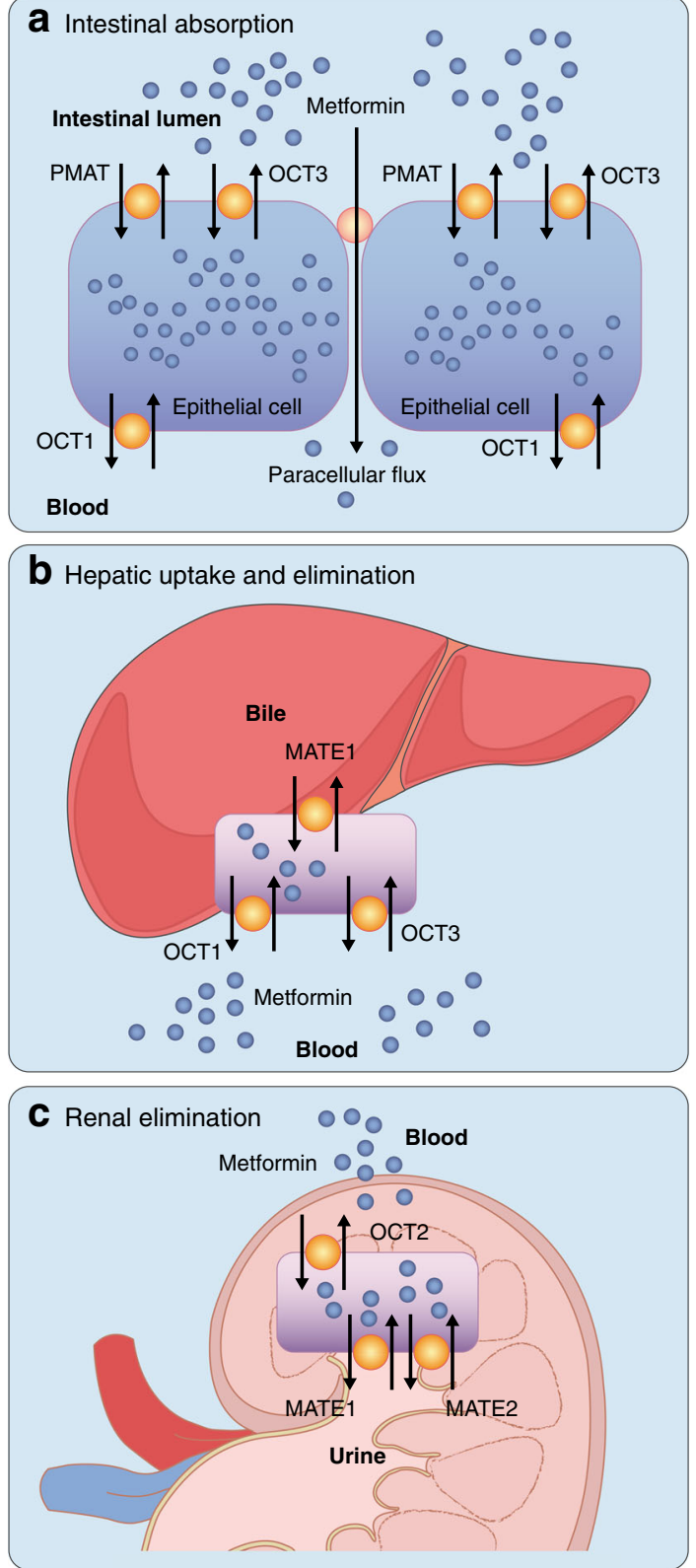

Fig. 1 Transporters responsible for the absorption and elimination of metformin into relevant tissues. Following ingestion, metformin reaches the gut endothelium (a), where it is taken up by enterocytes via PMAT and OCT3, located on the luminal surface of the gut epithelium. OCT1 transports metformin from the enterocyte to the serosal side of the endothelium. When it reaches the liver (b), OCT1 and OCT3 aid with metformin uptake into hepatocytes. Disposal of metformin into bile is facilitated by MATE1 and taken up by epithelial cells in the kidney (c) via OCT2, while MATE1 and MATE2 are involved in excretion of unchanged metformin into the urine, as it is not metabolised in humans. Figure adapted from [56]

response, namely initial $\mathrm{HbA}_{1 \mathrm{c}}$ reduction, odds of achieving a target $\mathrm{HbA}_{1 \mathrm{c}}$ of $<7 \%$, average $\mathrm{HbA}_{1 \mathrm{c}}$ on metformin monotherapy, or hazard of monotherapy failure, defined by the addition of a second agent by the treating clinician [41]. Whether this is a true negative result, or a false negative finding resulting from insufficient statistical power or the limitations inherent to observational studies (where treatment decisions are left to the discretion of the treating provider), is not clear. In the prospective South Danish Diabetes Study of 105 participants [42], both trough steady-state metformin concentrations and the change in $\mathrm{HbA}_{1 \mathrm{c}}$ after 6 months were lower with increasing number of reduced-function alleles at the four reduced-function variants, R61C, G401S, M420del and G465R [43]. A more recent study using radioisotope labelling has demonstrated that accumulation of metformin in human hepatocytes is diminished in carriers of the M420del and R61C variants in SLC22A1, without changes in circulating levels of the drug [44]. A study of side effects by the GoDARTS investigators in 251 metformin-intolerant individuals and 1915 metformin-tolerant individuals showed that the presence of two or more reduced-function alleles at R61C, C88R/rs55918055, G401S, M420del or G465R increased the odds of metformin intolerance by more than twofold, presumably by inducing accumulation of metformin in enterocytes [45]; the effect was additive with the concomitant use of OCT1-inhibiting medications, increasing the odds by fourfold. An initial report of association of the SLC22A1 intronic SNP rs622342 with metformin response in a retrospective Rotterdam study of 102 patients [46] has not been corroborated by subsequent independent publications [43, 47, 48]. Similarly, no associations have been found between reduced-function variants in OCT2 and metformin response [43, 47-49]. The Rotterdam group found an association of the intronic SNP rs2289669 in SLC47A1 (encoding MATE1) with metformin response, with the minor allele enhancing the reduction in $\mathrm{HbA}_{1 c}$; though this finding is supported by similar observations by the DPP [48] and Tkác et al [47], it was not seen in the South Danish Diabetes Study [43] nor in a large meta-analysis recently conducted by the Metformin Genetics (MetGen) Consortium [50]. In conclusion, the most solid evidence seems to support a role for coding missense variants in OCT1 with metformin response and/or intolerance.

\section{Second phase: GWAS}

The discovery and cataloguing of millions of SNPs across the human genome, the characterisation of their correlation structure across major ethnic groups, the manufacturing of high-throughput genotyping arrays, the development of statistical frameworks for rigorous association testing, and the assembly of human cohorts via international collaboration have contributed to ushering in the era of genome-wide association testing. An early collaboration by the GoDARTS and UK Prospective Diabetes Study (UKPDS) investigators led to the first GWAS for metformin response [21]. In a modest discovery sample of 1024 GoDARTS participants, an association signal with metformin response (defined as 
either reaching the clinical goal of $\mathrm{HbA}_{1 \mathrm{c}}<7 \%$ within 18 months of treatment initiation or a quantitative change in $\mathrm{HbA}_{1 \mathrm{c}}$ ) was detected around the gene encoding the ataxia-telangiectasia mutated kinase (ATM) (Fig. 2). The strongest association (odds of achieving the glycaemic target, 1.6) was detected at SNP rs 11212617 ; this signal was followed up in an independent set of 1783 GoDARTS and 1113 UKPDS participants, achieving genome-wide statistical significance. Other groups have reproduced this finding in participants with established type 2 diabetes [51]; however, the DPP has not been able to detect an association of this SNP with diabetes incidence or change in quantitative glycaemic traits in participants with impaired glucose tolerance [52], a failure that may be caused by insufficient statistical power or the intriguing possibility that pharmacogenetic interactions may diverge at different stages of the disease process [24].

The metformin pharmacogenetics field has coalesced to form the MetGen Consortium [53]. In a meta-analysis of 10,557 participants with a harmonised measure of metformin response, a genome-wide statistically significant association was observed at the SNP rs8192675, in an intron of the GLUT2 glucose transporter, encoded by $S L C 2 A 2$ and expressed in hepatocytes (Fig. 3) [54]. GLUT2 is an excellent biological candidate, in that the reduction in hepatic glucose output achieved by metformin is thought to be mediated by this transporter. Interestingly, the same allele that was associated with an improved response (lower on-treatment $\mathrm{HbA}_{1 \mathrm{c}}$ ) was also associated with a higher baseline $\mathrm{HbA}_{1 \mathrm{c}}$, consistent with a previously reported association of another SNP at this locus with fasting glucose in individuals without diabetes [55]. Hence, a larger effect was observed when on-treatment $\mathrm{HbA}_{1 \mathrm{c}}$ was not adjusted for baseline $\mathrm{HbA}_{1 \mathrm{c}}$, though the adjusted measure, while attenuated, retained genome-wide statistical significance. In support of the biological relevance of this variant, levels of expression of GLUT2 in the liver were also significantly associated with the same SNP (the allele associated with higher baseline $\mathrm{HbA}_{1 \mathrm{c}}$ was associated with lower expression of $S L C 2 \mathrm{A2}$ ). The magnitude of this association is deemed to be clinically relevant $\left(0.33 \%\right.$ absolute difference in $\mathrm{HbA}_{1 \mathrm{c}}$ between the two homozygous groups), in that it is comparable with thresholds commonly adopted by regulatory agencies to approve type 2 diabetes drugs. Similar to the ATM association, no association at this locus was found in the DPP, again raising the question of whether drug $\times$ gene interactions in the prediabetes state might differ from those in established type 2 diabetes, where organ deterioration may have already occurred.

\section{Conclusions and future directions}

To tailor the use of metformin to the most appropriate segment of the population, an improved understanding of its molecular mechanism of action is necessary. Given the documented heritability of metformin response in humans, agnostic genomic searches can facilitate both goals, but this requires the assembly of very large cohorts in order to detect likely modest effects. The MetGen Consortium represents one step in the right direction. Despite its most recent GWAS of $>10,000$ samples, the combined impact of the ATM and $S L C 2 A 2$ loci on metformin response was shown to be minimal, suggesting that many other genetic determinants of metformin action remain to be discovered, requiring much

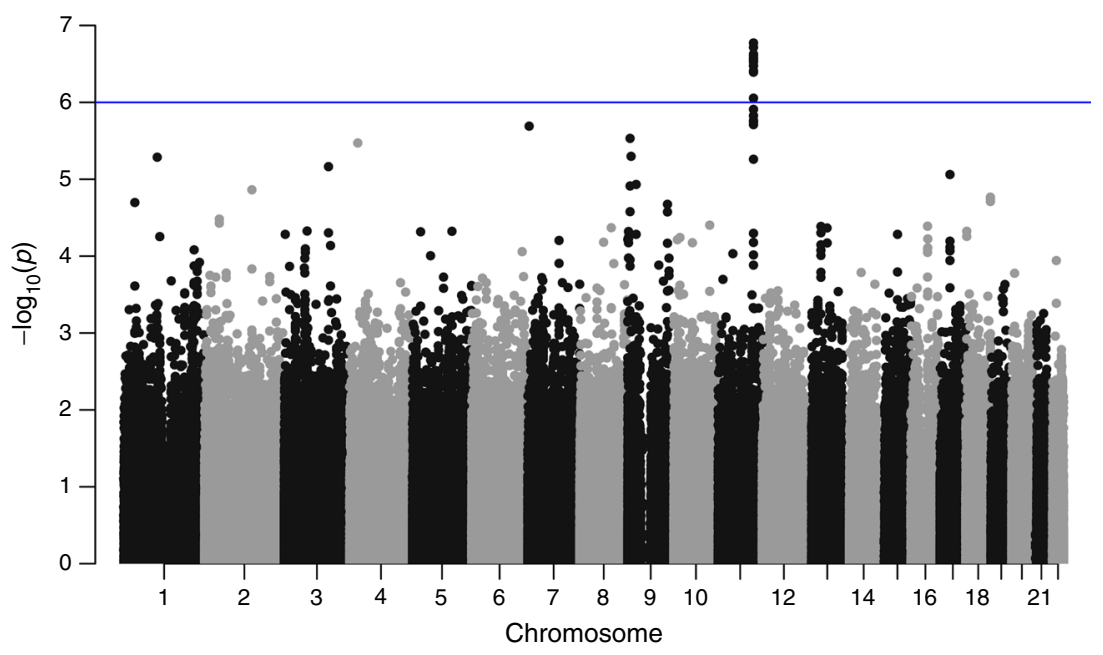

Fig. 2 Manhattan plot of the first GWAS for metformin response, indicating a single suggestive association signal in chromosome 11 near the ATM gene. Chromosomes are arranged linearly along the $x$-axis and denoted in different colours, with the negative $\log _{10}$ ( $p$ value) for association being shown along the $y$-axis. Each dot represents a single nucleotide polymorphism tested in the genome-wide array for association with metformin response, defined as reaching the clinical goal of $\mathrm{HbA}_{1 \mathrm{c}}$ $<7 \%$ within 18 months of treatment initiation. The blue line set at $p=1 \times 10^{-6}$ represents a threshold of suggestive statistical significance for evidence of association. Figure adapted by permission from Macmillan Publishers Ltd: Nature Genetics [21], copyright 2011 


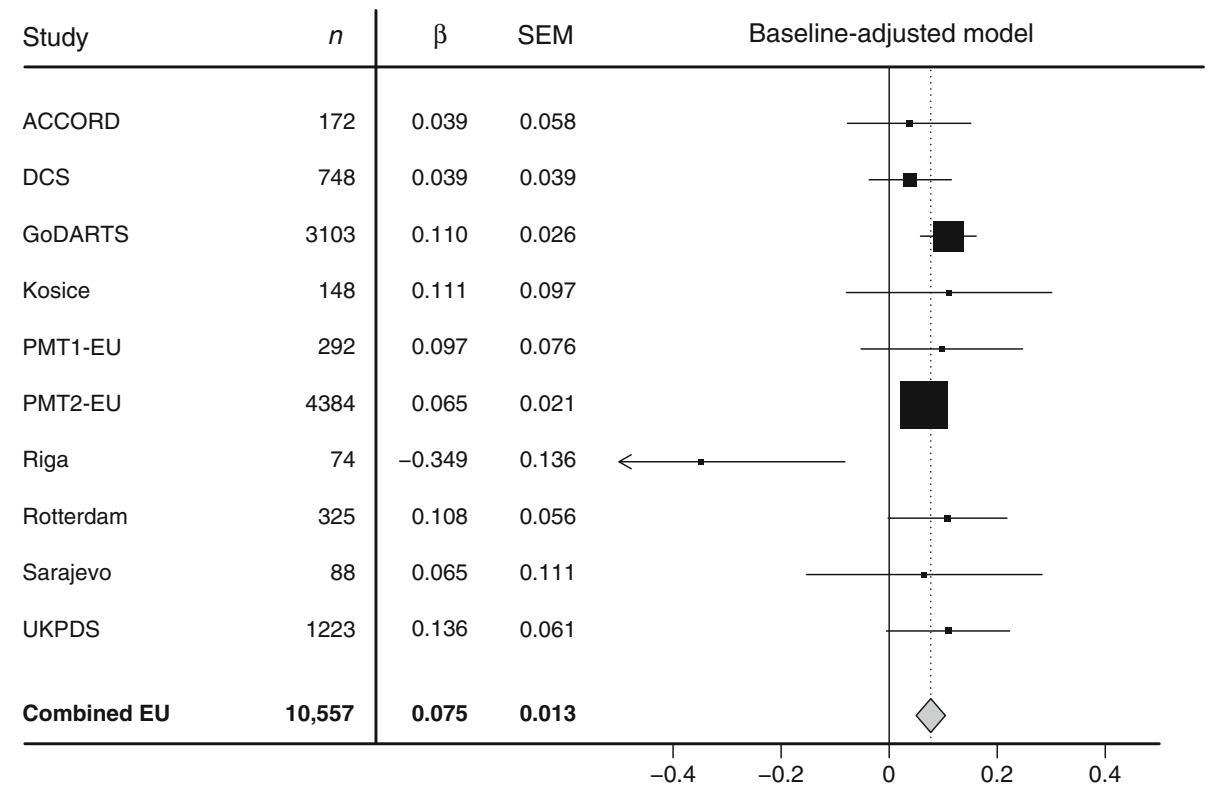

Fig. 3 Meta-analysis of the largest published GWAS for metformin response (defined as on-treatment $\mathrm{HbA}_{1 \mathrm{c}}$ adjusted for baseline $\mathrm{HbA}_{1 \mathrm{c}}$ ), comprising 10,557 participants of European descent. The summary result shows a genome-wide significant association at a SNP in the SLC2A2 gene, which encodes the GLUT2 glucose transporter expressed in

larger sample sizes and greater precision in pharmacogenetic measurements, such as drug doses, participant adherence and glycaemic response. However, most samples of participants treated with metformin and followed for glycaemic endpoints in a controlled fashion reside in the standard-of-care arms of randomised clinical trials for new type 2 diabetes agents, typically housed in the realm of pharmaceutical companies. Though non-disclosure by the pharmaceutical industry of pharmacogenetic outcomes for proprietary compounds might be defensible, it would be in everyone's best interest to share samples and data of individuals randomised to the active standard comparator (usually metformin), as the identification of metformin non-responders would open the door to a new patient population for whom newer agents could be marketed. While guarding against potential heterogeneity, a pharmacogenetic study that used every possible sample of a person treated with metformin, whether in healthcare systems, publicly funded studies or industry-sponsored clinical trials, would surely reveal new associations, leading to a richer understanding of metformin action, the potential design of superior agents, and better categorisation of patient subgroups based on their likelihood to benefit from this time-honoured treatment.

In practice, once robust pharmacogenetic associations are discovered and confirmed, they can be aggregated into genetic risk scores that explain a substantial proportion of the variance in glycaemic response or the appearance of side effects. They could be included into multi-trait genotyping arrays that hepatocytes. Adapted from Zhou et al, Nature Genetics (2016) [54]. ACCORD, Action to Control Cardiovascular Risk in Diabetes; DCS, Diabetes Cohort Study; PMT1/2-EU, Pharmacogenomics of Metformin Transporters project 1/2 (European descent)

capture all known clinically actionable genetic variants for most common diseases and approved drugs. This 'megachip' would only need to be deployed once in the lifetime of an individual at an affordable cost and the information could become part of that person's electronic medical record. Statistical algorithms that define the likelihood of response could be created, tested, refined and automatically triggered once a prescription order is initiated; decision support tools elaborated by experts would then inform the clinician, at the point of care, whether this patient is a good candidate for the selected agent. The methods and expertise exist to realise this vision but it will not come to fruition unless we generate the required knowledge base, the data stand rigorous scrutiny and cost-effectiveness analyses demonstrate that the benefit of clinical outcomes outweighs the expense incurred.

Funding JCF is a Massachusetts General Hospital Research Scholar. Work in the author's laboratory is supported by NIDDK R01 DK072041, NIDDK U01 DK105554, NIGMS R01 GM117163, NIDDK R01 DK105154, and NIDDK K24 DK110550.

Duality of interest JCF has received consulting honoraria from Merck, Boehringer-Ingelheim and Intarcia Therapeutics.

Contribution statement JCF conceived and wrote the entire manuscript, and is the sole contributor to this paper. 


\section{References}

1. Nathan DM, Buse JB, Davidson MB et al (2009) Medical management of hyperglycaemia in type 2 diabetes mellitus: a consensus algorithm for the initiation and adjustment of therapy: a consensus statement from the American Diabetes Association and the European Association for the Study of Diabetes. Diabetologia 52: $17-30$

2. Rodbard HW, Jellinger PS, Davidson JA et al (2009) Statement by an American Association of Clinical Endocrinologists/American College of Endocrinology consensus panel on type 2 diabetes mellitus: an algorithm for glycemic control. Endocr Pract 15:540559

3. Inzucchi SE, Bergenstal RM, Buse JB et al (2015) Management of hyperglycaemia in type 2 diabetes, 2015: a patient-centred approach. Update to a position statement of the American Diabetes Association and the European Association for the Study of Diabetes. Diabetologia 58:429-442

4. Bailey CJ, Turner RC (1996) Metformin. N Engl J Med 334:574 579

5. Nathan DM (2007) Finding new treatments for diabetes-how many, how fast... how good? N Engl J Med 356:437-440

6. The Diabetes Prevention Program Research Group (2002) Reduction in the incidence of type 2 diabetes with lifestyle intervention or metformin. N Engl J Med 346:393-403

7. Kirpichnikov D, McFarlane SI, Sowers JR (2002) Metformin: an update. Ann Intern Med 137:25-33

8. The United Kingdom Prospective Diabetes Study (1998) Effect of intensive blood-glucose control with metformin on complications in overweight patients with type 2 diabetes (UKPDS 34). Lancet 352:854-865

9. Knowler WC, Fowler SE, Hamman RF et al (2009) 10-year followup of diabetes incidence and weight loss in the Diabetes Prevention Program Outcomes Study. Lancet 374:1677-1686

10. Stumvoll M, Nurjhan N, Perriello G, Dailey G, Gerich JE (1995) Metabolic effects of metformin in non-insulin-dependent diabetes mellitus. N Engl J Med 333:550-554

11. The Diabetes Prevention Program Research Group (2012) Longterm safety, tolerability, and weight loss associated with metformin in the Diabetes Prevention Program Outcomes Study. Diabetes Care 35:731-737

12. Aroda VR, Knowler WC, Crandall JP, Group DPPR (2017) Metformin for diabetes prevention: insights gained from the Diabetes Prevention Program/Diabetes Prevention Program Outcomes Study. Diabetologia doi:10.1007/s00125-017-4361-9

13. Sam S, Ehrmann DA (2017) Metformin therapy for the reproductive and metabolic consequences of polycystic ovary syndrome. Diabetologia doi:10.1007/s00125-017-4306-3

14. Heckman-Stoddard B (2017) Repurposing metformin for the prevention of cancer and cancer recurrence. Diabetologia doi:10.1007/ s00125-017-4372-6

15. Turner RC, Cull CA, Frighi V, Holman RR (1999) Glycemic control with diet, sulfonylurea, metformin, or insulin in patients with type 2 diabetes mellitus: progressive requirement for multiple therapies (UKPDS 49). UK Prospective Diabetes Study (UKPDS) Group. JAMA 281:2005-2012

16. Kahn SE, Haffner SM, Heise MA et al (2006) Glycemic durability of rosiglitazone, metformin, or glyburide monotherapy. N Engl J Med 355:2427-2443

17. Zeitler P, Hirst K, Pyle L et al (2012) A clinical trial to maintain glycemic control in youth with type 2 diabetes. N Engl J Med 366: 2247-2256

18. Rena G, Hardie DG, Pearson ER (2017) The mechanisms of action of metformin. Diabetologia doi:10.1007/s00125-017-4342-Z
19. Williams LK, Padhukasahasram B, Ahmedani BK et al (2014) Differing effects of metformin on glycemic control by race-ethnicity. J Clin Endocrinol Metab 99:3160-3168

20. Lee SH, Wray NR, Goddard ME, Visscher PM (2011) Estimating missing heritability for disease from genome-wide association studies. Am J Hum Genet 88:294-305

21. GoDARTS and UKPDS Diabetes Pharmacogenetics Study Group, Wellcome Trust Case Control Consortium, Zhou K et al (2011) Common variants near ATM are associated with glycemic response to metformin in type 2 diabetes. Nat Genet 43:117-120

22. Yang J, Lee SH, Goddard ME, Visscher PM (2011) GCTA: a tool for genome-wide complex trait analysis. Am J Hum Genet 88:7682

23. Zhou K, Donnelly L, Yang J et al (2014) Heritability of variation in glycaemic response to metformin: a genome-wide complex trait analysis. Lancet Diabetes Endocrinol 2:481-487

24. Florez JC (2017) Pharmacogenetics in type 2 diabetes: precision medicine or discovery tool? Diabetologia 60:800-807

25. Graham GG, Punt J, Arora M et al (2011) Clinical pharmacokinetics of metformin. Clin Pharmacokinet 50:81-98

26. Zhou M, Xia L, Wang J (2007) Metformin transport by a newly cloned proton-stimulated organic cation transporter (plasma membrane monoamine transporter) expressed in human intestine. Drug Metab Dispos 35:1956-1962

27. Muller J, Lips KS, Metzner L, Neubert RH, Koepsell H, Brandsch M (2005) Drug specificity and intestinal membrane localization of human organic cation transporters (OCT). Biochem Pharmacol 70: $1851-1860$

28. Chen L, Pawlikowski B, Schlessinger A et al (2010) Role of organic cation transporter 3 (SLC22A3) and its missense variants in the pharmacologic action of metformin. Pharmacogenet Genomics 20: 687-699

29. Nies AT, Koepsell H, Winter S et al (2009) Expression of organic cation transporters OCT1 (SLC22A1) and OCT3 (SLC22A3) is affected by genetic factors and cholestasis in human liver. Hepatology 50:1227-1240

30. Shu Y, Sheardown SA, Brown C et al (2007) Effect of genetic variation in the organic cation transporter 1 (OCT1) on metformin action. J Clin Invest 117:1422-1431

31. Otsuka M, Matsumoto T, Morimoto R, Arioka S, Omote H, Moriyama Y (2005) A human transporter protein that mediates the final excretion step for toxic organic cations. Proc Natl Acad Sci U S A 102:17923-17928

32. Tanihara Y, Masuda S, Sato T, Katsura T, Ogawa O, Inui K (2007) Substrate specificity of MATE1 and MATE2-K, human multidrug and toxin extrusions $/ \mathrm{H}(+)$-organic cation antiporters. Biochem Pharmacol 74:359-371

33. Takane H, Shikata E, Otsubo K, Higuchi S, Ieiri I (2008) Polymorphism in human organic cation transporters and metformin action. Pharmacogenomics 9:415-422

34. Masuda S, Terada T, Yonezawa A et al (2006) Identification and functional characterization of a new human kidney-specific $\mathrm{H}+$ / organic cation antiporter, kidney-specific multidrug and toxin extrusion 2. J Am Soc Nephrol 17:2127-2135

35. Gong L, Goswami S, Giacomini KM, Altman RB, Klein TE (2012) Metformin pathways: pharmacokinetics and pharmacodynamics. Pharmacogenet Genomics 22:820-827

36. Kerb R, Brinkmann U, Chatskaia N et al (2002) Identification of genetic variations of the human organic cation transporter hOCT1 and their functional consequences. Pharmacogenetics 12:591-595

37. Leabman MK, Huang CC, Kawamoto M et al (2002) Polymorphisms in a human kidney xenobiotic transporter, OCT2, exhibit altered function. Pharmacogenetics 12:395-405

38. Sakata T, Anzai N, Shin HJ et al (2004) Novel single nucleotide polymorphisms of organic cation transporter 1 (SLC22A1) 
affecting transport functions. Biochem Biophys Res Commun 313: 789-793

39. Shu Y, Leabman MK, Feng B et al (2003) Evolutionary conservation predicts function of variants of the human organic cation transporter, OCT1. Proc Natl Acad Sci U S A 100:5902-5907

40. Shu Y, Brown C, Castro RA et al (2008) Effect of genetic variation in the organic cation transporter 1, OCT1, on metformin pharmacokinetics. Clin Pharmacol Ther 83:273-280

41. Zhou K, Donnelly LA, Kimber CH et al (2009) Reduced function SLC22A1 polymorphisms encoding organic cation transporter 1 (OCT1) and glycaemic response to metformin: a Go-DARTS study. Diabetes 58:1434-1439

42. Gram J, Henriksen JE, Grodum E et al (2011) Pharmacological treatment of the pathogenetic defects in type 2 diabetes: the randomized multicenter South Danish Diabetes Study. Diabetes Care 34:27-33

43. Christensen MM, Brasch-Andersen C, Green H et al (2011) The pharmacogenetics of metformin and its impact on plasma metformin steady-state levels and glycosylated hemoglobin A1c. Pharmacogenet Genomics 21:837-850

44. Sundelin EI, Gormsen LC, Jensen JB et al (2017) Genetic polymorphisms in organic cation transporter 1 attenuates hepatic metformin exposure in humans. Clin Pharmacol Ther doi:10.1002/cpt.701

45. Dujic T, Zhou K, Donnelly LA, Tavendale R, Palmer CN, Pearson ER (2015) Association of organic cation transporter 1 with intolerance to metformin in type 2 diabetes: a GoDARTS study. Diabetes 64:1786-1793

46. Becker ML, Visser LE, van Schaik RH, Hofman A, Uitterlinden AG, Stricker BH (2009) Genetic variation in the organic cation transporter 1 is associated with metformin response in patients with diabetes mellitus. Pharm J 9:242-247

47. Tkáč I, Klimcakova L, Javorsky M et al (2013) Pharmacogenomic association between a variant in SLC47A1 gene and therapeutic response to metformin in type 2 diabetes. Diabetes Obes Metab 15:189-191

48. Jablonski KA, McAteer JB, de Bakker PI et al (2010) Common variants in 40 genes assessed for diabetes incidence and response to metformin and lifestyle intervention in the diabetes prevention program. Diabetes 59:2672-2681

49. Shikata E, Yamamoto R, Takane H et al (2007) Human organic cation transporter (OCT1 and OCT2) gene polymorphisms and therapeutic effects of metformin. J Hum Genet 52:117-122

50. Dujic T, Zhou K, Yee SW et al (2017) Variants in pharmacokinetic transporters and glycaemic response to metformin: a MetGen metaanalysis. Clin Pharmacol Ther 101:763-772

51. van Leeuwen N, Nijpels G, Becker ML et al (2012) A gene variant near $A T M$ is significantly associated with metformin treatment response in type 2 diabetes: a replication and meta-analysis of five cohorts. Diabetologia 55:1971-1977

52. Florez JC, Jablonski KA, Taylor A et al (2012) The C allele of ATM rs11212617 does not associate with metformin response in the diabetes prevention Program. Diabetes Care 35:1864-1867

53. Pawlyk AC, Giacomini KM, McKeon C, Shuldiner AR, Florez JC (2014) Metformin pharmacogenomics: current status and future directions. Diabetes 63:2590-2599

54. Zhou K, Yee SW, Seiser EL et al (2016) Variation in the glucose transporter gene SLC2A2 is associated with glycemic response to metformin. Nat Genet 48:1055-1059

55. Dupuis J, Langenberg C, Prokopenko I et al (2010) New genetic loci implicated in fasting glucose homeostasis and their impact on type 2 diabetes risk. Nat Genet 42:105-116

56. Rena G, Pearson ER, Sakamoto K (2012) Molecular action and pharmacogenetics of metformin: current understanding of an old drug. Diabetes Manag 2:439-452 